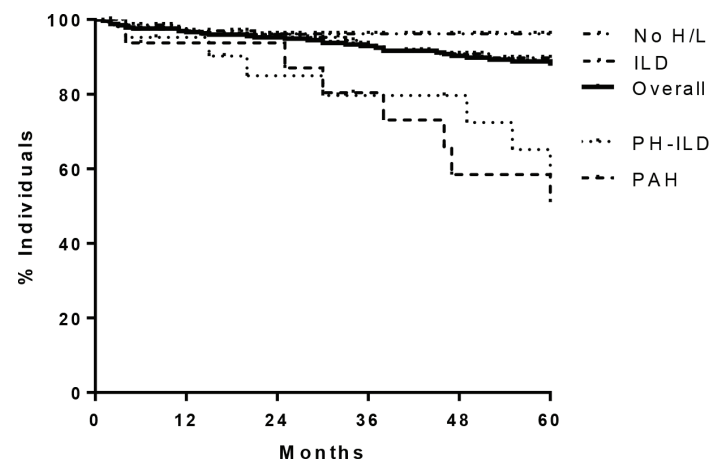

Abstract AB0640 Figure 1.. Kaplan-Meier curves for 5-years survival in SSc patients (Logrank 33.40 , p 0 001) Abbreviations: No H/L: absence of heart or lung involvement; ILD: interstitial lung disease; PH-ILD: Pulmonary hypertension associated to ILD; PAH: pulmonary arterial hypertension.

Table I. Statistically significant prognostic factors for 5 -years mortality at univariate and multivariate analysis (Cox proportional hazards modelling).

\begin{tabular}{lcccccc}
\hline & \multicolumn{3}{c}{ UNIVARIATE ANALYSIS } & \multicolumn{3}{c}{ MULTIVARIATE ANALYSIS } \\
\hline & HR & $95 \%$ IC & $P$ & HR & $95 \%$ IC & $P$ \\
\hline dcSSc & 3.45 & $1.33-8.95$ & 0.01 & 4,02 & $1.86-8.71$ & 0.0001 \\
Age at diagnosis & 2.63 & $1.01-6.75$ & 0.04 & 2.71 & $1.12-6.61$ & 0.03 \\
Delay in referral & 2.64 & $1.00-6.84$ & 0.05 & 2.73 & $1.11-6.7$ & 0.03 \\
BMI & 0.90 & $0.82-0.99$ & 0.03 & 0.91 & $0.84-0.98$ & 0.02 \\
No H/L Involvement & 0.45 & $0.21-0.97$ & 0.04 & 0.17 & $0.06-0.5$ & 0.001 \\
\hline
\end{tabular}

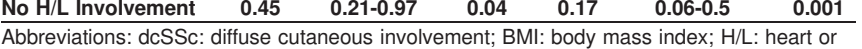
lung involvement; HR: hazard risk.

Disclosure of Interests: Fabio Cacciapaglia: None declared, Marco Fornaro: None declared, Fabio Montini: None declared, Livio Urso: None declared, Sergio Colella: None declared, Laura Coladonato: None declared, Maria Grazia Anelli: None declared, Emanuela Praino: None declared, Giuseppe Lopalco Speakers bureau: SOBI, BMS, Florenzo lannone Consultant for: $F$ lannone has received consultancy fees and/or speaker honoraria from Pfizer, AbbVie, MSD, BMS, Novartis, Lilly, UCB outside this work, Speakers bureau: $F$ lannone has received consultancy fees and/or speaker honoraria from Pfizer, AbbVie, MSD, BMS, Novartis, Lilly, UCB outside this work

DOI: 10.1136/annrheumdis-2019-eular.5058

\section{AB0641 VITAMIN D SERUM LEVELS AND THE RISK OF DIGITAL ULCERS IN SYSTEMIC SCLEROSIS: A LONGITUDINAL STUDY}

Cristian Caimmi, Eugenia Bertoldo, Alice Pozza, Paola Caramaschi, Giovanni Orsolini, Davide Gatti, Maurizio Rossini, Ombretta Viapiana. Rheumatology Unit, University of Verona, Verona, Italy

Background: Vitamin D is known to influence the immune system in many different diseases although longitudinal studies on systemic sclerosis (SSc) are still missing.

Objectives: this study wants to investigate if variations in vitamin $D$ serum levels (25OHD) over time affect digital ulcers (DU) in SSc.

Methods: this is a retrospective study on 65 patients. Data on disease characteristics and 25OHD were collected in 2011 and 2016.

Results: The mean age of our cohort was 58 (12) years with a mean disease duration of 9.5 (5.3) years. Most of our patients had a limited subset (69.2\%). At baseline $50.8 \%$ and $41.5 \%$ after 5 years had $25 \mathrm{OHD}$ $<30 \mathrm{ng} / \mathrm{mL}$. Supplemented patients (8750 IUlweek) at baseline were 39 $(60.0 \%)$ and $45(69.2 \%)$ at the end of follow up. Nevertheless, 31 $(47.7 \%)$ had a decrease of $25 \mathrm{OHD}$ in 5 years. In univariate analysis, patients with incident digital ulcers (DU) had a decrease in 25OHD as compared to patients with no incident DU (-17.4 (37.0) vs. 13.0 (89.5), $\mathrm{p}=0.018$ ). No differences in $25 \mathrm{OHD}$ variations were found for other disease characteristics. In multivariate analysis correcting for previous DU and mRSS at baseline, patients with a decrease in 25OHD had an increased risk of developing DU (OR (IC95\%): 16.6 (1.7 to 164.5), $\mathrm{p}=0.017)$.
Conclusion: In this study we have shown that a decrease in 250HD increases the risk of developing DUs and that vitamin $D$ supplementation with the doses currently recommended may be insufficient in SSc. Further studies in wider cohorts are needed to confirm these results and to evaluate the effectiveness of a more aggressive or different vitamin $D$ supplementation.

Disclosure of Interests: Cristian Caimmi: None declared, Eugenia Bertoldo: None declared, Alice Pozza: None declared, Paola Caramaschi: None declared, Giovanni Orsolini Speakers bureau: Grunenthal, Davide Gatti Speakers bureau: Abiogen, Amgen, Janssen-Cilag, Mundipharma, Pfeizer, Maurizio Rossini: None declared, Ombretta Viapiana Speakers bureau: Novartis, Abbvie, Eli-Lilly, Sanofi Genzyme DOI: 10.1136/annrheumdis-2019-eular.5318

\section{AB0642 DESCRIPTION OF SAE1/2 ANTIBODY IN A DERMATOMYOSITIS COHORT}

Jordi Camins-Fàbregas ${ }^{1}$, Clara Esteve-Cols ${ }^{2}$, Ivette Casafont-Solé ${ }^{1}$ Melania Martínez-Morillo ${ }^{1}$, Maria Aparicio-Espinar ${ }^{1}$, Lourdes Mateo ${ }^{1}$ Anne Riveros ${ }^{1}$, Laia Gifre ${ }^{1}$, Clara Sangüesa-Gomez ${ }^{1}$, Águeda Prior-Español ${ }^{1}$, Annika Nack ${ }^{1}$, Anahy Brandy-Garcia ${ }^{1}$, Monica Munera-Campos ${ }^{3}$, Isabel Bielsa-

Marsol $^{3}$, Eva Martinez-Cáceres ${ }^{1}$, Alejandro Olive ${ }^{1}$, Bibiana Quirant ${ }^{2}$,

Susana Holgado ${ }^{1,2} .{ }^{1}$ Hospital Universitari Germans Trias i Pujol, Rheumatology, Badalona, Spain; ${ }^{2}$ Hospital Universitari Germans Trias i Pujol, Immunology, Badalona, Spain; ${ }^{3}$ Hospital Universitari Germans Trias i Pujol, Dermatology, Badalona, Spain

Background: Myositis specific antibodies have gained special importance in last years. Its knowledge has allowed stratifying patients in different clinical phenotypes, predicting with greater accuracy prognosis and establishing a clinical attitude to follow. SAE1/2 antibody (anti-small ubiquitinlike modifier activating enzyme) was first described in 2007 in patients with amyopathic dermatomyositis with cutaneous and digestive involvement. Its prevalence ranges from $8 \%$ in European cohorts to $3 \%$ in Asians.

Objectives: Describing myositis specific antibodies (MSA) in a cohort of inflammatory myopathies. To characterize the clinical phenotype of SAE1/ 2 antibody and to compare it with the rest of MSA.

Methods: Patients diagnosed of dermatomyositis in a tertiary hospital from 1978-2018, according to the criteria of Bohan and Peter (1975) and according to Dalakas classification criteria (2015). Clinical and analytical data, including the immunological profile were collected, as well as the treatments received and the evolution of the disease.

Results: Out of 46, 41 dermatomyositis had positive antinuclear antibodies (ANA). $55 \%$ percent had ANA titles $\geq 640$, being the fine speckled pattern the most frequent $(43 \%)$ followed by coarse speckled and homogeneous (13.6\% each).

$72 \%$ had MSA, the most frequent being antiJo1 (27.3\%) followed by MDA5 (18.2\%) and SAE1/2 (15\%). Up to $40 \%$ had two or more antibodies, being the association with antibodies Ro52 and Ro60, the most frequent.

5 patients presented positivity against SAE antibody. In comparison to the rest of MSA, $80 \%$ presented with cutaneous debut $(p=0.00)$, being the most frequent manifestations heliotrope erythema $(p=0.00)$, Gottron papules $(p=0.10)$ and skin rash $(p=0.00) .60 \%$ had pathological capillaroscopy compared to $15 \%(p=0.00)$. Muscular balance was preserved in $60 \%$ of patients. Sixty percent had dysphagia vs $9 \%(p=0.00)$. Two of them had lung involvement (alveolar hemorrhage and rapidly progressive pneumonitis). In comparison with SAE negative group, patients presented more pulmonary hypertension $(44.5 \mathrm{vs} 34 \mathrm{mmHg})$, without reaching significant differences. No significant differences were found between muscle enzyme levels' neither acute phase reactants.

As complications, one patient presented a myocarditis with quickly rapidly progressive pneumonitis that required high doses of corticotherapy and another one alveolar hemorrhage, which was treated the same way. All patients required corticotherapy at doses of $\mathrm{mg} / \mathrm{kg}$, requiring two of them to be treated with DMARDs (methotrexate and dolquine) and one of them with immunoglobulins due to cutaneous involvement.

Mortality rate was $40 \%$ due to rapidly progressive lung affectation and cardiorespiratory arrest.

Conclusion: Higher prevalence of SAE1/2 antibody in our cohort may be explained due to the use of amplified myositis antibodies kit that specifically includes this antibody. These patients typically present with cutaneous involvement and dysphagia but also lung affectation as a complication. Studies with larger samples should be performed in order to know the prognosis of this antibody specificity.

Disclosure of Interests: None declared

DOI: 10.1136/annrheumdis-2019-eular.3325 